\title{
EVIDENCE OF VERY LOW METALLICITY AND HIGH IONIZATION STATE IN A STRONGLY LENSED, STAR-FORMING DWARF GALAXY AT $z=3.417^{*}$
}

\author{
R. Amorín ${ }^{1,3}$, A. Grazian ${ }^{1}$, M. Castellano ${ }^{1}$, L. Pentericci $^{1}$, A. Fontana ${ }^{1}$, \\ V. Sommariva ${ }^{1}$, A. VAn der Wel ${ }^{2}$, M. Maseda $^{2}$, and E. Merlin ${ }^{1}$ \\ ${ }^{1}$ INAF-Osservatorio Astronomico di Roma, via Frascati 33, I-00040 Monteporzio Catone, \\ Roma, Italy; ricardo.amorin@oa-roma.inaf.it \\ 2 Max-Planck-Institut für Astronomie, Königstuhl 17, D-69117 Heidelberg, Germany \\ Received 2014 February 7; accepted 2014 March 23; published 2014 May 21
}

\begin{abstract}
We investigate the gas-phase metallicity and Lyman continuum (LyC) escape fraction of a strongly gravitationally lensed, extreme emission-line galaxy at $z=3.417, J 1000+0221 S$, recently discovered by the CANDELS team. We derive ionization- and metallicity-sensitive emission-line ratios from $H+K$ band Large Binocular Telescope (LBT)/LUCI medium resolution spectroscopy. $J 1000+0221 S$ shows high ionization conditions, as evidenced by its enhanced $[\mathrm{O} \mathrm{III}] /\left[\mathrm{O}_{\mathrm{II}}\right]$ and $[\mathrm{O} \mathrm{III}] / \mathrm{H} \beta$ ratios. Strong-line methods based on the available line ratios suggest that $J 1000+0221 S$ is an extremely metal-poor galaxy, with a metallicity of $12+\log (\mathrm{O} / \mathrm{H})<7.44\left(Z<0.05 Z_{\odot}\right)$, placing it among the most metal-poor star-forming galaxies at $z \gtrsim 3$ discovered so far. In combination with its low stellar mass $\left(2 \times 10^{8} M_{\odot}\right)$ and high star formation rate $\left(5 M_{\odot} \mathrm{yr}^{-1}\right)$, the metallicity of $J 1000+0221 S$ is consistent with the extrapolation of the mass-metallicity relation traced by Lyman-break galaxies at $z \gtrsim 3$ to low masses, but it is 0.55 dex lower than predicted by the fundamental metallicity relation at $z \lesssim 2.5$. These observations suggest a rapidly growing galaxy, possibly fed by massive accretion of pristine gas. Additionally, deep LBT/LBC photometry in the $U G R$ bands are used to derive a limit to the LyC escape fraction, thus allowing us to explore for the first time the regime of sub- $L^{*}$ galaxies at $z>3$. We find a $1 \sigma$ upper limit to the escape fraction of $23 \%$, which adds a new observational constraint to recent theoretical models predicting that sub- $L^{*}$ galaxies at high- $z$ have high escape fractions and thus are the responsible for the reionization of the universe.
\end{abstract}

Key words: galaxies: abundances - galaxies: dwarf - galaxies: evolution - galaxies: fundamental parameters galaxies: high-redshift - galaxies: starburst - gravitational lensing: strong

Online-only material: color figures

\section{INTRODUCTION}

Recently, van der Wel et al. (2013, hereafter vdW13) presented the serendipitous discovery of the first strong galaxy lens at $z_{\text {lens }}>1, J 100018.47+022138.74$. This quadrupole lens system was found in the COSMOS field covered by the CANDELS (Grogin et al. 2011; Koekemoer et al. 2011) survey. Using Hubble Space Telescope (HST) near-infrared (NIR) imaging from CANDELS and NIR spectroscopy from the Large Binocular Telescope (LBT), the authors reported a record lens redshift $z_{L}=1.53 \pm 0.09$ and a strongly magnified $(40 \times)$ source at redshift $z_{S}=3.417 \pm 0.001$ (hereafter $J 1000+0221 S$ ). While the lens is a quiescent and relatively massive galaxy, the magnified source was found to be a low-mass $\left(M_{\star} \sim 10^{8} M_{\odot}\right)$, extreme emission-line galaxy (EELG) with unusually high rest-frame [O III] $\lambda 5007$ equivalent width $\left(\mathrm{EW}_{0} \sim 1000 \AA\right)$.

The scarcity of strong galaxy lenses at high redshift makes the discovery of $J 100018.47+022138.74$ especially remarkable. Strikingly enough, the probability of finding an EELG being lensed by another galaxy appears to be very low, unless these galaxies become significantly more abundant at high $z$ (vdW13). Consistently, a large number of low-mass EELGs at $z \sim 2$ have started to emerge from deep surveys (e.g., van der Wel et al. 2011; Atek et al. 2011; Guaita et al. 2013; Maseda et al. 2013, 2014) and recent observational evidences point to their ubiquity at $z \sim 5-7$ (Smit et al. 2014). Low-mass

\footnotetext{
* Based on Large Binocular Telescope (LBT) observations.

3 ASTRODEEP fellow.
}

galaxies with extreme nebular content at lower redshift are mostly chemically unevolved systems, characterized by their compactness, high specific star formation rates (SFRs), high ionization, and low metallicities, which make them lie offset from the main sequence of galaxies in fundamental scaling relations between mass, metallicity, and SFR (e.g., Amorín et al. 2010, 2014b; Atek et al. 2011; Nakajima \& Ouchi 2013; Ly et al. 2014). At high redshift, however, the full characterization of these properties in intrinsically faint galaxies requires enormous observational effort (e.g., Erb et al. 2010; Maseda et al. 2013) and detailed studies are mostly restricted to those sources being subject to strong magnification by gravitational lensing (e.g., Fosbury et al. 2003; Richard et al. 2011; Christensen et al. 2012; Brammer et al. 2012; Belli et al. 2013; Bayliss et al. 2013).

The aim of this Letter is to fully characterize the lensed EELG $J 1000+0221 S$ at $z=3.417$ presented by vdW13. This unique galaxy will help us to investigate two key issues. Using the deepest available LBT photometry and spectroscopy we will first derive robust estimates of the ionization and metallicity properties of $J 1000+0221 S$ through strong emission-line ratios. This provides additional hints on the evolutionary stage of the galaxy and allows us to add new constrains to the low-mass end of the mass-metallicity-SFR relation at $z \sim 3.4$. Finally, $J 1000+0221 S$ will give the opportunity to derive, for the first time, a limit to the Lyman continuum (LyC) escape fraction at $z>3$ in the sub- $L^{*}$ regime, as suggested by Vanzella et al. (2012). 


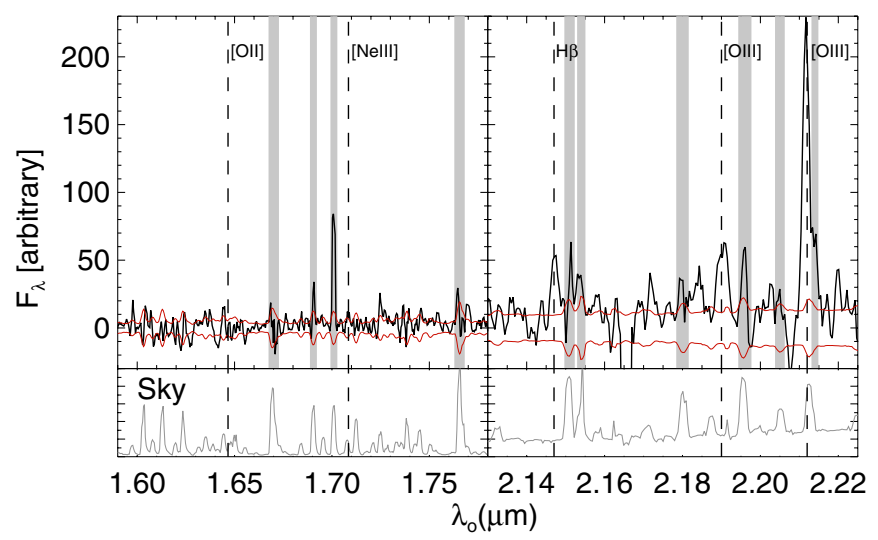

Figure 1. LUCI/LBT $H+K$ spectrum of $J 1000+0221$ smoothed with the instrumental resolution. We show overplotted in red the $3 \sigma$ noise spectrum. The position of both detected and undetected bright nebular emission lines are indicated by dashed lines. Shaded vertical regions show spectral regions affected by strong sky emission lines.

(A color version of this figure is available in the online journal.)

\section{SPECTROSCOPIC DATA AND MEASUREMENTS}

In order to derive line ratios for $J 1000+0221 S$, we re-analyze the LBT/LUCI NIR spectrum presented by vdW13 and shown in Figure 1. It consists of a $3 \mathrm{hr}$ sequence of individual, dithered $120 \mathrm{~s}$ exposures using the $H+K$ grism in a $1^{\prime \prime}$ wide slit and seeing of $\sim 0$.' 6 . The spectrum was calibrated in wavelength and corrected by telluric absorption, but an absolute flux calibration was not possible (see vdW13). Full details on the reduction procedure will be discussed in a forthcoming paper (Maseda et al. 2014).

Alternatively, we determine a relative flux calibration between the spectra coming from the two grisms, $H$ and $K$, after computing the integral flux of the two spectral ranges and their corresponding color $(H-K)_{\text {spec }}$. Then, we compare $(H-K)_{\text {spec }}$ and the analog color, $(H-K)_{\text {phot }}$, derived from the NEWFIRM Medium Band Survey multiwavelength catalog (Whitaker et al. 2011). We find the difference between the two measurements $\sim 10 \%$, so we conclude that uncertainties due to flux calibration in the derived flux ratios involving lines in the blue and red parts of the spectrum are not significant.

Integrated fluxes and uncertainties for the three emission lines detected in the $K$ spectrum, namely $\mathrm{H} \beta$, and [O III] $\lambda \lambda 4958$, 5007 , were measured using the IRAF task splot by summing all pixels of the emission line after linear subtraction of the continuum. Uncertainties are derived following Amorín et al. (2010). We do not detect any line above $3 \sigma$ in the $H$-band spectrum. However, it is still possible to place stringent upper limits on the [O II] and [Ne III] emission lines of $F_{l}<5 \sigma_{l}$, where $\sigma_{l}$ is the integral rms noise of the spectrum over the total velocity width expected for the line. For the latter we assume the average width of the detected [O III] lines (13 $\AA$ ).

Emission-line ratios are computed after de-reddening the derived fluxes and upper limits using the Calzetti et al. (2000) extinction law. Apart from $\mathrm{H} \beta$, no other Balmer lines are detected in the spectrum due to sensitivity limits. Thus, based on the stellar reddening derived by vdW13, $E(B-V)_{\star}=0_{-0.0}^{+0.2}$, we assume here $E(B-V)_{\text {gas }}=0.2\left(=1 \sigma\right.$ uncertainty in $\left.E(B-V)_{\star}\right)$, consistent with the mean $E(B-V)_{\text {gas }}$ found for low-mass galaxies of similar EW[O III] in Amorín et al. (2012, 2014a, 2014b). Clearly, by using a higher extinction we derive even more conservative upper limits on metallicity. However, we note
Table 1

Main Derived Properties of $J 100018.47+022138.74$

\begin{tabular}{lccc}
\hline \hline R.A. $(J 2000)$ & 150.07697 & $z$ & 3.417 \\
Decl. $(J 2000)$ & +2.36076 & {$[\mathrm{O}$ II $] \lambda \lambda 3727,3729 / \mathrm{H} \beta$} & $<0.30$ \\
$M_{\mathrm{B}}$ & $-17.8 \pm 0.3$ & {$[\mathrm{O}$ III $] \lambda 4959 / \mathrm{H} \beta$} & $1.44 \pm 1.35$ \\
$E(B-V)_{\star}$ & $0.0_{-0.0}^{+0.2}$ & {$[\mathrm{O}$ III $] \lambda 5007 / \mathrm{H} \beta$} & $4.47 \pm 1.25$ \\
$\log M_{\star}\left[M_{\odot}\right]$ & $8.41_{-0.30}^{+0.25}$ & {$[\mathrm{Ne}$ III $] \lambda 3868 / \mathrm{H} \beta$} & $<0.20$ \\
SFR $\left[M_{\odot} \mathrm{yr}^{-1}\right]$ & $5 \pm 2$ & $12+\log (\mathrm{O} / \mathrm{H})$ & $<7.44_{-0.17}^{+0.20}$ \\
\hline
\end{tabular}

Notes. $B$-band absolute magnitude, stellar reddening, star formation rate, and stellar mass were derived from the spectral energy distribution (SED) fitting after correction for magnification (van der Wel et al. 2013). Line fluxes are given relative to $F(\mathrm{H} \beta)=1$.

that a factor of two higher extinction should not significantly affect our results.

\section{EMISSION LINE AND METALLICITY PROPERTIES}

In Table 1 we present the emission-line ratios and upper limits relative to $\mathrm{H} \beta$ derived for $J 1000+0221 S$, along with other physical properties taken from vdW13. The available line ratios are used in Figure 2 to study the ionization and metallicity properties of $J 1000+0221 S$ through different diagnostic diagrams. In particular, the $[\mathrm{O} \mathrm{III}] / \mathrm{H} \beta$ and $[\mathrm{O} \mathrm{II}] / \mathrm{H} \beta$ line ratios suggest that $J 1000+0221 S$ is a high excitation, low-metallicity galaxy. Consequently, the remarkably low [O II]/[O III] ratio suggests an unusually high ionization. This is confirmed by the high ionization parameter, $\log q_{\text {ion }}>8.5 \mathrm{~cm} \mathrm{~s}^{-1}$, derived from the ionizationsensitive ratio $[\mathrm{O} \mathrm{II}] /[\mathrm{O} \mathrm{III}]$ and adopting the calibration based on photoionization models by Levesque \& Richardson (2014). Being higher than those found in normal galaxies, the derived $q_{\text {ion }}$ is only comparable to those found in other EELGs (e.g., Nakajima \& Ouchi 2013; Ly et al. 2014; Amorín et al. 2014a, 2014b).

Strong telluric absorption makes it impossible to detect the faint $T_{e}$-sensitive auroral line [O III] $\lambda 4363$, precluding a direct determination of the oxygen abundance. Alternatively, we use the set of collisionally excited emission lines available for $J 1000+0221 S$ to derive its metallicity following a strong-line method based on the $R 23$ (三 $([\mathrm{O}$ II $]+[\mathrm{O}$ III) $] / \mathrm{H} \beta$ ) parameter, which shows a well-known bi-valued relation with $Z$. In particular, here we use the calibration and the method proposed and explained in detail by Maiolino et al. (2008). The use of this calibration gives us the possibility of comparing our results with other Lyman-break galaxies (LBGs) at similar redshift (e.g., Troncoso et al. 2014).

In short, this method combines the calibration of $R 23$ and other nebular line ratios as a function of metallicity, as shown in Figure 2 . In the low-metallicity regime $\left(Z \lesssim 0.5 Z_{\odot}\right)$ these relations are calibrated using local galaxies with direct $\left(T_{e}\right)$ metallicities, while at higher $Z$ they are based on photoionization models. This method has the advantage that at least two of the relations involved increase monotonically with $Z$, so they can remove the degeneracy in the $R 23-Z$ relation. In particular, $J 1000+0221 S$ shows a remarkably low [O II] upper limit, which makes any diagnostic based on such a line, e.g., $[\mathrm{O}$ II $] / \mathrm{H} \beta$ versus $Z$ or [O III]/[O II] versus $Z$ in Figure 2, almost conclusive that this object is extremely metal-poor. In practice, we solve the calibrations shown in Figure 2 simultaneously and iteratively. An uncertainty of a factor of two in the adopted $E(B-V)_{\text {gas }}$ has been propagated to the error bars shown in Figure 2. Finally, the best metallicity limit that satisfies the various emission-line ratios is obtained by adopting the lower $R 23$ branch, resulting 

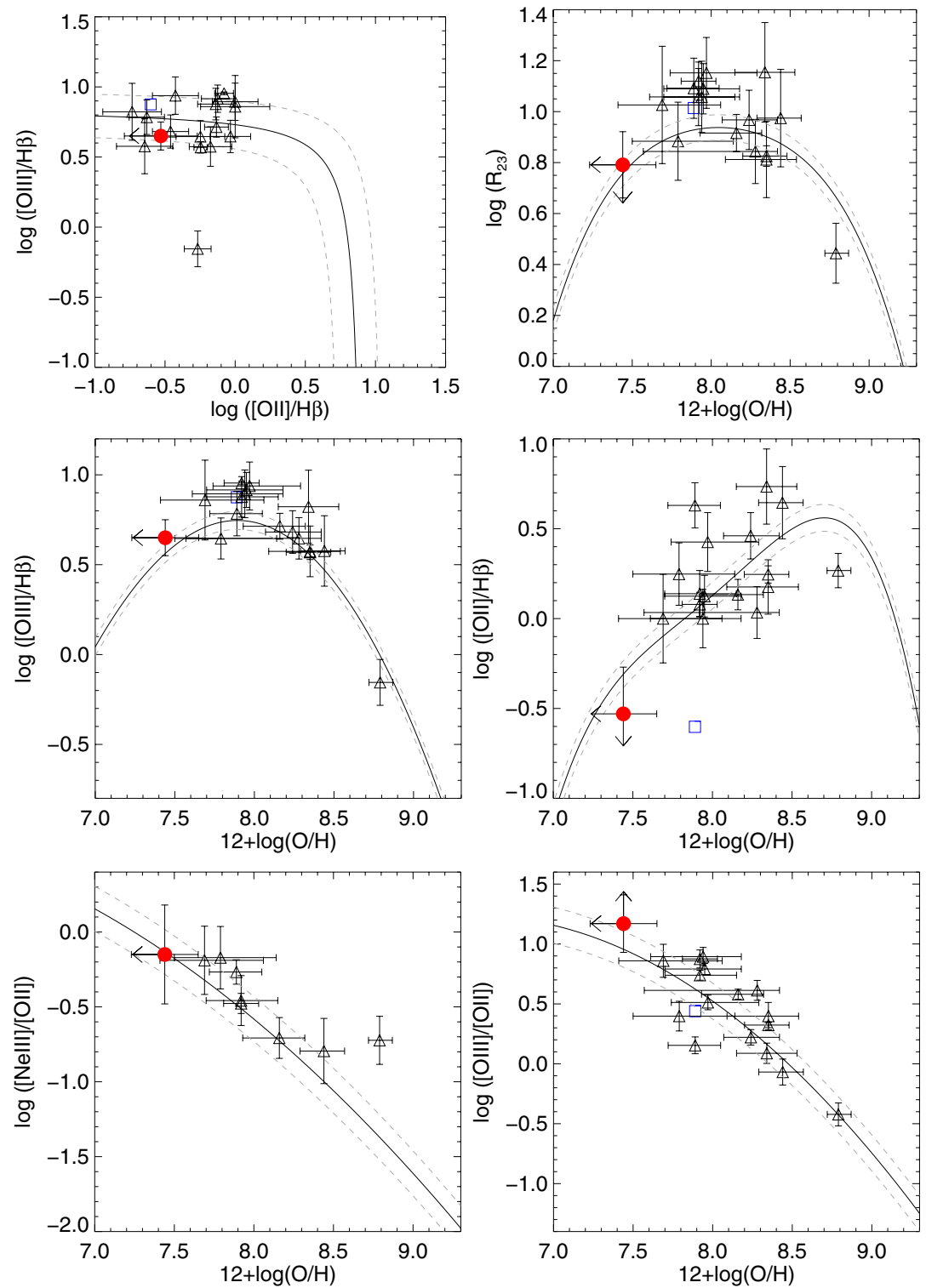

Figure 2. Diagnostic diagrams and strong-line metallicity calibrations from Maiolino et al. (2008, see also Nagao et al. 2006). Gray dashed lines are $1 \sigma$ uncertainties for these relations. The best metallicity limit for $J 1000+0221 S$ that satisfies the various emission-line ratios is obtained by adopting the lower $R 23$ branch, as shown by the red circle. LBGs at $z \geqslant 3$ from the AMAZE (Maiolino et al. 2008) and LSD (Mannucci et al. 2009) samples (see also Troncoso et al. 2014, triangles) and the strongly lensed Lynx galaxy (Fosbury et al. 2003, blue square) are shown for comparison.

(A color version of this figure is available in the online journal.)

in an upper limit in metallicity $12+\log (\mathrm{O} / \mathrm{H})<7.44 \pm 0.2$ at the $68 \%$ confidence level.

\section{DISCUSSION}

\subsection{The Low-mass End of the Mass-Metallicity Relation at $z \sim 3.4$}

At redshift $z \sim 2-3$, where massive galaxies are rapidly assembling most of their present-day stellar mass (Hopkins \& Beacom 2006), the mass-metallicity relation (MZR) traced by luminous LBGs shows significant evolution (Erb et al. 2006; Maiolino et al. 2008; Mannucci et al. 2009; Troncoso et al. 2014). However, due to the challenge that measuring metallicities represents and poor statistics, the shape and normalization of the MZR at $z \gtrsim 3$ are still poorly constrained, especially in its low-mass end. Thus it is particularly interesting to study the position of $J 1000+0221 S$ in the MZR.
In Figure 3 we use our metallicity limit and the stellar mass ${ }^{4}$ derived by vdWell 13 to show the good agreement found between the position of $J 1000+0221 S$ and the extrapolation to low stellar masses of the MZR traced by more massive LBGs at $z \gtrsim 3$. Compared to the other few galaxies of similar or slightly higher masses and redshift, the upper limit on the metallicity of $J 1000+0221 S$ is $\sim 0.5$ dex lower.

The scatter and normalization of the MZR at low $z$ have been associated with the star formation activity and with the presence of intense gas flows in a tight relation among mass, metallicity, and SFR, the so-called "fundamental metallicity

\footnotetext{
4 Stellar masses have been derived following Finkelstein et al. (2012) by fitting models accounting for nebular (line plus continuum) emission to the lens-subtracted SED of the source in four HST bands, which correspond to rest-frame UV. Photometry at longer wavelengths (optical rest-frame) were not included as source plus lens emission could not be deblended. However, we note that the sum of the best-model luminosities in the optical (rest-frame) for lens and source appears consistent, being only slightly lower than the total observed luminosities after de-magnification (see Figure 3 in vdW13).
} 

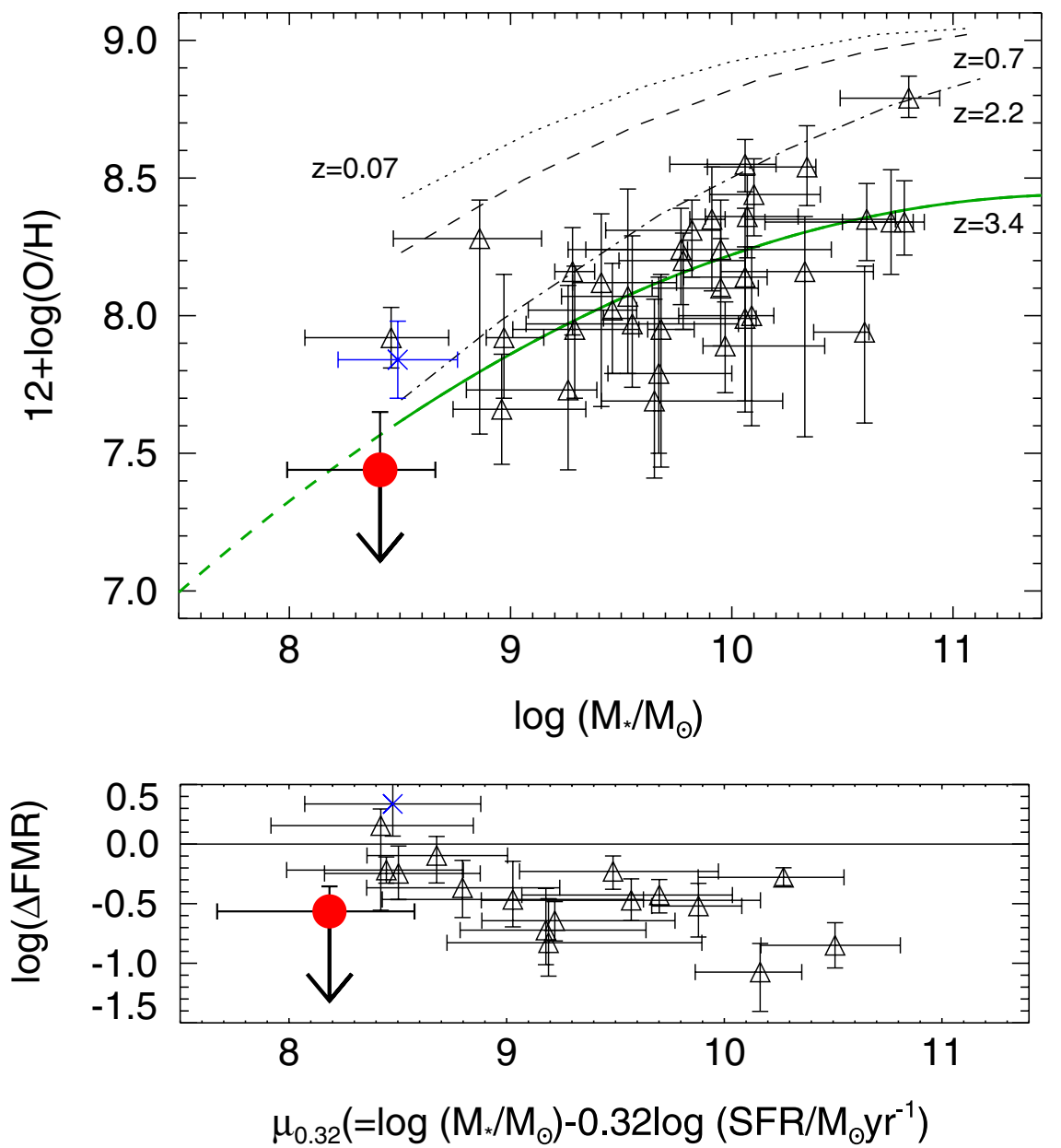

Figure 3. Upper panel: mass-metallicity relation at $z \geqslant 3$. Bottom panel: deviation in metallicity $\left(\Delta \mathrm{FMR}=Z_{\mathrm{obs}}-Z_{\mathrm{FMR}}\right)$ from the FMR (Mannucci et al. 2011$)$ as a function of $\mu_{32}$. Dotted, dashed, dot-dashed, and solid lines in the upper panel show the MZR at $z \sim 0.07,0.7,2.2$, and 3.4, respectively, presented by Troncoso et al. (2014). The solid line in the lower panel indicates a perfect match between the measured metallicities and the ones derived through the FMR. LBGs from Troncoso et al. (2014, triangles) and the low-mass lensed galaxy "the Sextet" at $z=3.04$ from Belli et al. (2013, blue cross) are shown for comparison.

(A color version of this figure is available in the online journal.)

relation" (FMR; Mannucci et al. 2010). According to the FMR, at a given stellar mass, galaxies with higher SFR do have lower metallicities (see also Pérez-Montero et al. 2013). In contrast to the MZR, the FMR has been found to persist in galaxies out to redshift $z \sim 2.5$ (Belli et al. 2013). However, at $z \gtrsim 3$ most LBGs studied so far, e.g., those in the AMAZE and LSD samples, are found to be more metal-poor than predicted by the FMR. This may suggest a change in the mechanisms that give rise to the FMR or a strong selection effect at these redshifts (Troncoso et al. 2014). Alternatively, it may suggest that metallicity calibrations based on local galaxies could not apply to high- $z$ galaxies due to their comparatively higher excitation/ionization conditions (Kewley et al. 2013). Also, recent studies on local galaxies using integral field spectroscopy have questioned the validity of the FMR as due to aperture biases (Sánchez et al. 2013).

In Figure 3, we reproduce the results by Troncoso et al. (2014) for the FMR and include the position of $J 1000+0221 S$ using the extrapolation of the FMR to low stellar masses by Mannucci et al. (2011). Clearly, the metallicity of $J 1000+0221 S$ is at least 0.55 dex lower than predicted by the FMR.

The very low metallicity, high specific SFR $\left(\sim 10^{-8} \mathrm{yr}^{-1}\right)$, and extremely high EWs of $J 1000+0221 S$ are indicative of a rapidly growing galaxy in an early stage of its evolution.
The offset position found in the MZR and FMR by at least 1 dex and 0.5 dex, respectively, compared to the local relations suggests the action of massive gas flows (e.g., Dayal et al. 2013). One interesting possibility is that the recent star formation in $J 1000+0221 S$ is being fed by the massive accretion of pristine gas in the cold-gas flow mode, as predicted by cosmological simulations to be the main mode of building up galaxies at these redshifts (e.g., Dekel et al. 2009) and supported by observational evidences in some low-metallicity starbursts (Cresci et al. 2010; Sanchez Almeida et al. 2014).

\subsection{The First Estimation of the Lyman Continuum Escape Fraction in the Very Low Luminosity Regime $L=0.04 L^{*}$}

The discovery of $J 1000+0221 S$ also offers the great opportunity to derive one of the first limits to the LyC escape fraction at $z>3$ in a regime of very low intrinsic luminosity $\left(L \sim 0.05 L^{*}\right)$, as suggested by Vanzella et al. (2012). The photometry of this object has been derived by Boutsia et al. (2014) from deep LBC data in the $U G R$ filters used in that work to search for LBGs at $2.7 \leqslant z \leqslant 3.4$. This lensed galaxy has $\mathrm{AB}$ magnitudes $R=24.15 \pm 0.02, G=25.22 \pm 0.04$ and an upper limit $U \geqslant 28.93$ at $1 \sigma$. Note that this galaxy was not selected as an LBG candidate in Boutsia et al. (2014) due to its color $G-R=1.07$, which is slightly redder than the typical color 
cut $G-R \leqslant 1$ adopted. This is due to the fact that, as shown in $\mathrm{vdW13}$, the spectral energy distribution (SED) of this galaxy is contaminated by the lens galaxy, an elliptical at $z=1.53$.

Using the LBC photometry alone, we can derive a limit to the LyC escape fraction for the lensed galaxy, adopting the same technique already used in Boutsia et al. (2011). To get rid of the light contamination by the elliptical $z=1.53$, we have checked the photometry in the Advanced Camera for Surveys band $V_{606}$, which is the closest $H S T$ band to our $R$ filter. The total magnitude of the lens + source system is $V_{606}=24.15$, equal to our $R$-band magnitude. Thus we can safely assume that the contribution by the lens corresponds to $R=26.4$ and the corrected magnitude of $J 1000+0221 S$ is $R=24.3$. Adopting the upper limit in the $U$ band as an estimate for the maximum flux emitted by the lensed source and using the corrected $R$-band flux, we derive the relative escape fraction simply from

$$
f_{\mathrm{esc}}^{\mathrm{rel}}=\frac{L_{1500} / L_{900}}{\text { flux }_{R} / \operatorname{flux}_{U}} \exp \left(\tau_{\mathrm{IGM}}\right) .
$$

As in Boutsia et al. (2011), we adopt a value of three as an estimate for the intrinsic $L_{1500} / L_{900}$ ratio. Following Prochaska et al. (2009) we derive a correction for the intergalactic medium (IGM) transmission of $\exp \left(-\tau_{\mathrm{IGM}}\right)=0.1811$ at the redshift of the source, $z=3.417$. We thus obtain an upper limit to the escape fraction of $23.2 \%$ at $1 \sigma$ confidence level. While this limit is not so stringent with respect to other estimates in the literature at $z=3$ (e.g., Boutsia et al. 2011; Vanzella et al. 2010; Mostardi et al. 2013), it is nonetheless important since we are probing an intrinsic luminosity regime still unexplored before this work. The source magnitude corrected for lensing is $R=28.3$, corresponding to an absolute magnitude of -17.4 or equivalently to $L_{1500}=0.036 L^{*}(z=3)$, assuming a typical value $L^{*}(z=3)=-21.0$. This is equivalent to a depth of 32.9 mag in the $U$ band, after correcting our upper limit for the magnification factor.

A number of theoretical models (e.g., Nakajima \& Ouchi 2013; Paardekooper et al. 2013; Ferrara \& Loeb 2013; Dijkstra et al. 2014) are investigating the processes involved during the end of the so-called "Dark Ages." The main stream under all these models is that the sources responsible for re-ionizing the universe are dwarf galaxies (sub- $L^{*}$ ) at $z \sim z_{\text {reion }} \sim 7$. Some of these works also assume LyC escape fractions greater than 30\%-50\% even at lower redshifts (e.g., Nakajima \& Ouchi 2013; Dijkstra et al. 2014).

Due to IGM absorption increasing with redshift, the LyC escape fraction can only be directly measured up to $z \sim 3$. For this reason our limit on the $f_{\text {esc }}$ from an ultra-faint $z=3.4$ galaxy provides an interesting input to re-ionization model predictions, under the assumption that faint galaxies such as $J 1000+0221 S$ are representative of the whole faint galaxy population at $z>3$. In fact, our stringent $23 \%$ limit is at the lowest boundary of the range $f_{\text {esc }} \sim 20 \%-30 \%$ which, according to recent observational evidence (Finkelstein et al. 2012; Grazian et al. 2012), allows star-forming galaxies to keep the IGM ionized at $z>6$.

Our current results for $J 1000+0221 S$ highlight the strength of strong lensing techniques to study the properties of lowmass star-forming galaxies at $z \gtrsim 3$. If, as expected, analogs of $J 1000+0221 S$ are ubiquitous at higher redshifts, forthcoming data from the CANDELS survey and the HST Frontier Fields Initiative and its spectroscopic follow-ups, will likely provide a statistically significant number of such systems, which is needed to derive more stringent limits to the escape fraction of $\mathrm{LyC}$ photons by ultra-faint galaxies and to study in greater detail the mechanisms driving the early phases of galaxy formation.

We thank the referee for helpful suggestions that contributed to the improvement of this manuscript. This work is partially founded by the FP7 SPACE project ASTRODEEP (reference No. 312725), supported by the European Commission. We thank Roberto Maiolino for helpful assistance with metallicity determinations.

Facilities: HST (ACS, WFPC3), KPNO (NEWFIRM), LBT (LUCI), LBT (LBC)

\section{REFERENCES}

Amorín, R., Pérez-Montero, E., Contini, T., et al. 2014a, A\&A, submitted (arXiv:1403.3441)

Amorín, R., Pérez-Montero, E., Vílchez, J. M., \& Papaderos, P. 2012, ApJ, 749,185

Amorín, R., Sommariva, V., Castellano, M., et al. 2014b, A\&A, submitted (arXiv:1403.3692)

Amorín, R. O., Pérez-Montero, E., \& Vílchez, J. M. 2010, ApJL, 715, L128

Atek, H., Siana, B., Scarlata, C., et al. 2011, ApJ, 743, 121

Bayliss, M. B., Rigby, J. R., Sharon, K., et al. 2013, ApJ, submitted (arXiv:1310.6695)

Belli, S., Newman, A. B., \& Ellis, R. S. 2013, ApJ, 772, 141

Boutsia, K., Grazian, A., Giallongo, E., et al. 2011, ApJ, 736, 41

Boutsia, K., et al. 2014, A\&A, 563, A142

Brammer, G. B., Sánchez-Janssen, R., Labbé, I., et al. 2012, ApJL, 758, L17

Calzetti, D., Armus, L., Bohlin, R. C., et al. 2000, ApJ, 533, 682

Christensen, L., Richard, J., Hjorth, J., et al. 2012, MNRAS, 427, 1953

Cresci, G., Mannucci, F., Maiolino, R., et al. 2010, Natur, 467, 811

Dayal, P., Ferrara, A., \& Dunlop, J. S. 2013, MNRAS, 430, 2891

Dekel, A., Birnboim, Y., Engel, G., et al. 2009, Natur, 457, 451

Dijkstra, M., Wyithe, S., Haiman, Z., Mesinger, A., \& Pentericci, L. 2014 MNRAS, in press (arXiv:1401.7676)

Erb, D. K., Pettini, M., Shapley, A. E., et al. 2010, ApJ, 719, 1168

Erb, D. K., Shapley, A. E., Pettini, M., et al. 2006, ApJ, 644, 813

Ferrara, A., \& Loeb, A. 2013, MNRAS, 431, 2826

Finkelstein, S. L., Papovich, C., Ryan, R. E., et al. 2012, ApJ, 758, 93

Fosbury, R. A. E., Villar-Martín, M., Humphrey, A., et al. 2003, ApJ, 596, 797

Grazian, A., Castellano, M., Fontana, A., et al. 2012, A\&A, 547, A51

Grogin, N. A., Kocevski, D. D., Faber, S. M., et al. 2011, ApJS, 197, 35

Guaita, L., Francke, H., Gawiser, E., et al. 2013, A\&A, 551, A93

Hopkins, A. M., \& Beacom, J. F. 2006, ApJ, 651, 142

Kewley, L. J., Dopita, M. A., Leitherer, C., et al. 2013, ApJ, 774, 100

Koekemoer, A. M., Faber, S. M., Ferguson, H. C., et al. 2011, ApJS, 197, 36

Levesque, E. M., \& Richardson, M. L. A. 2014, ApJ, 780, 100

Ly, C., Malkan, M. A., Nagao, T., et al. 2014, ApJ, 780, 122

Maiolino, R., Nagao, T., Grazian, A., et al. 2008, A\&A, 488, 463

Mannucci, F., Cresci, G., Maiolino, R., Marconi, A., \& Gnerucci, A. 2010, MNRAS, 408, 2115

Mannucci, F., Cresci, G., Maiolino, R., et al. 2009, MNRAS, 398, 1915

Mannucci, F., Salvaterra, R., \& Campisi, M. A. 2011, MNRAS, 414, 1263

Maseda, M., et al. ApJ, submitted

Maseda, M. V., van der Wel, A., da Cunha, E., et al. 2013, ApJL, 778, L22

Mostardi, R. E., Shapley, A. E., Nestor, D. B., et al. 2013, ApJ, 779, 65

Nagao, T., Maiolino, R., \& Marconi, A. 2006, A\&A, 459, 85

Nakajima, K., \& Ouchi, M. 2013, MNRAS, in press (arXiv:1309.0207)

Paardekooper, J.-P., Khochfar, S., \& Dalla Vecchia, C. 2013, MNRAS, 429, L94

Pérez-Montero, E., Contini, T., Lamareille, F., et al. 2013, A\&A, 549, A25

Prochaska, J. X., Worseck, G., \& O’Meara, J. M. 2009, ApJL, 705, L113

Richard, J., Jones, T., Ellis, R., et al. 2011, MNRAS, 413, 643

Sanchez Almeida, J., Morales-Luis, A. B., Munoz-Tunon, C., et al. 2014, ApJ, 783,45

Sánchez, S. F., Rosales-Ortega, F. F., Jungwiert, B., et al. 2013, A\&A, 554, A58 Smit, R., Bouwens, R. J., Labbe, I., et al. 2014, ApJ, 784, 58

Troncoso, P., Maiolino, R., Sommariva, V., et al. 2014, A\&A, 563, A58 van der Wel, A., Straughn, A. N., Rix, H.-W., et al. 2011, ApJ, 742, 111 van der Wel, A., van de Ven, G., Maseda, M., et al. 2013, ApJL, 777, L17 Vanzella, E., Giavalisco, M., Inoue, A. K., et al. 2010, ApJ, 725, 1011 Vanzella, E., Nonino, M., Cristiani, S., et al. 2012, MNRAS, 424, L54 Whitaker, K. E., Labbé, I., van Dokkum, P. G., et al. 2011, ApJ, 735, 86 